\title{
NEW PREFERENCE VIOLATION INDICES FOR THE CONDITION OF ORDER PRESERVATION
}

\author{
JiŘÍ MAZUREK*
}

\begin{abstract}
Consistency of pairwise comparisons is one particular aspect that is studied thoroughly in the recent decades. However, since the introduction of the concept of the condition of the order preservation in 2008, there is no inconsistency measure based on the aforementioned condition. Therefore, the aim of this paper is to fill this gap and propose new preference violation indices for measuring violation of the condition of the order preservation. Further, an axiomatic system for the proposed measures is discussed, and it is shown that the proposed indices satisfy uniqueness, invariance under permutation, invariance under inversion of preferences and continuity axioms.
\end{abstract}

Mathematics Subject Classification. 03F25, 90B50.

Received March 9, 2020. Accepted January 11, 2022.

\section{INTRODUCTION}

Over last decades, pairwise comparisons became one of the most popular tools in multiple criteria decision making. Probably the most known methods incorporating pairwise comparisons with hundreds of applications are the analytic hierarchy (network) process (AHP/ANP), PROMETHEE, and ELECTRE, see e.g. Behzadian et al. [7], Govindan and Jepsen [34], and Vaidya and Kumar [60].

In recent years, a number of studies focused on the problem of inconsistency of pairwise comparisons, properties of inconsistency indices and their relationships, as well as on proposition of axiomatic systems for inconsistency indices, see e.g. Alonso and Lamata [1], Bozóki and Rapcsák [10], Bozóki et al. [11], Brunelli et al. [17], Brunelli [14,15], Brunelli and Fedrizzi [16], Csató [23-25], Golden and Wang [33] Holsztynski and Koczkodaj [35], Koczkodaj [38, 39], Koczkodaj and Szwarc [40], Koczkodaj et al. [42, 43], Kulakowski and Talaga [45], Mazurek and Perzina [50], Mazurek [47,48], Peláez and Lamata [52], Ramík [56], Saaty [57,58], or Saaty [59].

The condition of the order preservation $(\mathrm{COP})$ in the context of (multiplicative) pairwise comparisons was introduced in 2008 by Bana e Costa and Vansnick [2]. The COP constitutes an alternative approach to the examination of the (in)consistency of pairwise comparison matrices. The usual notion of (multiplicative) consistency requires that if an object $\mathrm{A}$ is 3 times better than an object $\mathrm{B}$, and $\mathrm{B}$ is 2 times better than an object C, then A should be exactly 6 times better than $\mathrm{C}$, and this relationship should hold for every triple (A, B, C) of compared objects. However, decision makers are seldom precise to such an extent, especially when the number of compared objects is high. Moreover, even objective databases, for example from sports, suffer from

Keywords. Pairwise comparisons, condition of order preservation, inconsistency, preference violation index.

Silesian University in Opava, Opava, Czech Republic.

*Corresponding author: mazurek@opf.slu.cz 
inconsistencies, see e.g. Bozóki et al. [12], Chao et al. [19], Csató [20], Csató [21], Csató and Tóth [29], or Petróczy [54].

The condition of order preservation focuses on the relationship between direct comparison of two (four) objects, and their weights. If an object A is directly preferred over $\mathrm{B}$, then also the weight of A should be greater than the weight of the object B. This condition is called the Preservation of Order Preference condition (POP).

Further, when comparing $\mathrm{A}$ with $\mathrm{B}$ and $\mathrm{C}$ with $\mathrm{D}$, if $\mathrm{A}$ is more preferred over $\mathrm{B}$ than $\mathrm{C}$ is preferred to $\mathrm{D}$, then also the ratio of weights of $\mathrm{A}$ and $\mathrm{B}$ should be greater than the ratio of weights of $\mathrm{C}$ and $\mathrm{D}$. The condition is called the Preservation of Order of Intensity Preference condition (POIP). Together, the POP and POIP conditions form the COP condition

Since its introduction in 2008, several papers on the COP appeared, see e.g. Cavallo and D'Apuzzo [18], Kulakowski [44], or Kulakowski et al. [46]. These studies focused on finding sufficient (or neccessary) conditions for the COP satisfaction. In study Mazurek and Ramík [51], a new method for a priority vector elicitation was proposed in a form of non-linear programming problem.

However, what is missing in the literature on the COP so far, is a measure of inconsistency in the COP framework. Therefore, the aim of the paper is to fill this research gap by proposing new preference violation indices based on the condition of the order preservation. Also, an axiomatic system for the proposed measures is discussed.

The paper is organized as follows: Section 2 provides a brief introduction to pairwise comparisons, in Section 3 the condition of order preservation is introduced, and new preference violation indices are proposed in Section 4. A numerical example is provided in Section 5 and an axiomatic system for preference violation indices is discussed in Section 6. Monte Carlo simulations are provided in Section 7, and Conclusions close the article.

\section{Preliminaries}

\subsection{Pairwise comparisons method}

The input data for the PC method is a PC matrix $A=\left[a_{i j}\right]$, where $a_{i j} \in \mathbb{R}_{+}$and $i, j \in\{1, \ldots, n\}$. The values of $a_{i j}$ and $a_{j i}$ indicate the relative importance (or preference) of objects $i$ and $j$.

Definition 2.1. A matrix $A$ is said to be (multiplicatively) reciprocal if:

$$
\forall i, j \in\{1, \ldots, n\}: a_{i j}=1 / a_{j i}
$$

and $A$ is said to be (multiplicatively) consistent if:

$$
\forall i, j, k \in\{1, \ldots, n\}: a_{i j} \cdot a_{j k} \cdot a_{k i}=1 .
$$

Since human judgment is subjective, a pairwise comparison matrix may be (and often is) inconsistent. Therefore, many inconsistency indices were proposed in the literature, and their properties had been discussed, see e.g. Alonso and Lamata [1], Brunelli et al. [17], Brunelli [14,15], Brunelli and Fedrizzi [16], Csató [24,25], Koczkodaj [38], Koczkodaj and Szwarc [40], Mazurek [47], or Peláez and Lamata [52].

The result of the pairwise comparisons method is a priority vector (vector of weights) $w=\left(w_{1}, \ldots, w_{n}\right)$ that assigns positive values (weights) $w_{i}$ to each of the $n$ compared objects. According to the EVM (eigenvalue method) proposed by Saaty, see Saaty $[58,59]$, vector $w$ is determined as the rescaled principal eigenvector of $A$. Thus, assuming that $A w=\lambda_{\max } w$ the priority vector $w$ is

$$
w=\gamma\left[w_{1}, \ldots, w_{n}\right]^{T}
$$

where $\gamma$ is a scaling factor. Usually it is assumed that $\gamma=\left[\sum_{i=1}^{n} w_{i}\right]^{-1}$. 
In the geometric mean method (GMM) the weight of $i$ th alternative is given by the geometric mean of the $i$ th row of $A$. Thus, the priority vector is given as:

$$
w=\gamma\left[\left(\prod_{r=1}^{n} a_{1 r}\right)^{\frac{1}{n}}, \ldots,\left(\prod_{r=1}^{n} a_{n r}\right)^{\frac{1}{n}}\right]^{T}
$$

where $\gamma$ is a scaling factor again.

There are numerous inconsistency indices that measure inconsistency of pairwise comparison matrices, such as Saaty's consistency index CI and consistency ratio CR, Koczkodaj's inconsistency index KI, and many others, see e.g. Alonso and Lamata [1], Barzilai [4,15], Brunelli and Fedrizzi [16], Golden and Wang [33], Koczkodaj [38], or Peláez and Lamata [52].

\section{THE CONDITION OF ORDER PRESERVATION}

The condition of order preservation (COP) provides an alternative approach to the evaluation of (multiplicative) inconsistency of pairwise comparisons. The standard notion of inconsistency is provided by relation (2.2). However, this relation is not relevant to the COP. In the COP theoretical framework, relation (2.2) is replaced by the following two relations.

Definition 3.1. Let $A=\left[a_{i j}\right]$ be a pairwise comparison matrix, and let $w=\left(w_{1}, \ldots, w_{n}\right)$ be a priority vector associated to $A$. A PC matrix $A$ is said to satisfy the Preservation of Order Preference condition (POP) with respect to the priority vector $w$ if

$$
a_{i j}>1 \Rightarrow w_{i}>w_{j} \quad \text { for all } i, j .
$$

Definition 3.2. Let $A=\left[a_{i j}\right]$ be a pairwise comparison matrix, and let $w=\left(w_{1}, \ldots, w_{n}\right)$ be a priority vector associated to $A$. A PC matrix $A$ is said to satisfy the Preservation of Order of Intensity Preference condition (POIP) with respect to the priority vector $w$ if

$$
a_{i j}>1, a_{k l}>1 \text {, and } a_{i j}>a_{k l} \Rightarrow \frac{w_{i}}{w_{j}}>\frac{w_{k}}{w_{l}} \quad \text { for all } i, j, k, l .
$$

We say that a matrix $A=\left[a_{i j}\right]$ is the POP consistent, if it satisfies Definition 3.1, and the POIP consistent, if it satisfies Definition 3.2, respectively. We say that a matrix $A=\left[a_{i j}\right]$ is the COP consistent if it is both POP and POIP consistent.

Remark 3.3. Let $A=\left[a_{i j}\right]$ be a pairwise comparison matrix considered consistent according to relation (2.2), and let $w=\left(w_{1}, \ldots, w_{n}\right)$ be a priority vector associated to $A$ via the eigenvalue method or the geometric mean method. Then it is obvious that POP and POIP conditions are satisfied, but the reverse statement is not true in general. Further on, POP and POIP conditions are satisfied for a consistent matrix according to relation (2.2) if a method used for a priority vector derivation satisfies the axiom called correctness, see e.g. Barzilai et al. [6], Barzilai [3], Csató [26], or Fichtner [31].

To discriminate between the two concepts of inconsistency, the approach based on relation (2.2) can be considered "numerical consistency", while the approach based on relations ((3.1) and (3.2)) can be regarded as a "preferential consistency". In addition, we reserve the term "inconsistency index" to the numerical consistency, and we use the term "preference violation index" for an inconsistency measure in preferential consistency framework to avoid confusion.

\section{Measuring violation of the COP}

Almost all inconsistency indices introduced in the literature in the context of numerical consistency, with the exception of Saaty's indices CI and CR, are based on relation (2.2). Since relation (2.2) is not relevant to the preferential consistency, a preference violation index for the preferential consistency has to be based on relations (3.1) and (3.2), and is introduced in the following subsections. 


\subsection{Violation frequency index}

One possible way of evaluating satisfaction (or violation, respectively) of the condition of order preservation is to evaluate how many individual POP and POIP conditions are satisfied or violated. For a pairwise comparison matrix of the order $n$ one must check $\left(n^{2}-n\right)$ individual POP conditions (relations (3.1)) and $\left[\left(n^{2}-n\right)\left(n^{2}-n-2\right)\right]$ individual POIP conditions (relations (3.2)). However, since $a_{i j}>1$ implies $a_{j i}<1$, there are at most $\max _{(\mathrm{POP})}=\frac{1}{2}\left(n^{2}-n\right)$ individual $\mathrm{POP}$ conditions and at most $\max _{(\mathrm{POIP})}=$ $\frac{1}{4}\left[\left(n^{2}-n\right)\left(n^{2}-n-2\right)\right]$ individual POIP conditions that can be failed. We say that a pairwise comparison matrix satisfies the POP condition if all individual POP conditions hold. The same applies to the POIP condition. This line of reasoning leads to the following two-dimensional violation frequency index (VFI):

Definition 4.1. Let $A=\left[a_{i j}\right]$ be a PC matrix, and let $w=\left(w_{1}, \ldots, w_{n}\right)$ be the vector of weights associated with the matrix $A=\left[a_{i j}\right]$ via the geometric mean method. Further, let $n_{1}$ and $n_{2}$ denote the number of failed POP and POIP individual conditions respectively. Then the violation frequency index (VFI) is given as follows:

$$
\operatorname{VFI}_{n}(A)=\left[\alpha=\frac{n_{1}}{\max _{(\mathrm{POP})}}, \beta=\frac{n_{2}}{\max _{(\mathrm{POIP})}}\right]
$$

where $\alpha \in[0,1]$ expresses the ratio of failed individual POP conditions and $\beta \in[0,1]$ expresses the ratio of failed individual POIP conditions.

\subsection{Preference violation indices}

First, let relax the strict inequalities in definitions of the POP and the POIP conditions (relations (3.1) and (3.2)) in the following way, which is more convenient for the evaluation of inconsistency:

$$
\begin{aligned}
a_{i j}>1 & \Rightarrow w_{i} \geq w_{j} \\
a_{i j}>1, a_{k l}>1, \text { and } a_{i j}>a_{k l} & \Rightarrow \frac{w_{i}}{w_{j}} \geq \frac{w_{k}}{w_{l}} .
\end{aligned}
$$

The following definitions introduce two preference violation indices for the POP (index Alpha) and POIP (index Beta) conditions, respectively.

Definition 4.2. Let $A=\left[a_{i j}\right]$ be a PC matrix, and let $w=\left(w_{1}, \ldots, w_{n}\right)$ be the vector of weights associated with the matrix $A$ via the geometric mean method. Then the preference violation index $\operatorname{Alpha}(A, w)$ for the POP condition is given as follows:

$$
\operatorname{Alpha}(A, w)=\sum_{I(1)}\left(w_{j}-w_{i}\right)
$$

By analogy, the preference violation index $\operatorname{Beta}(A, w)$ for the POIP condition is given as:

$$
\operatorname{Beta}(A, w)=\sum_{I(2)}\left(\frac{w_{k}}{w_{l}}-\frac{w_{i}}{w_{j}}\right)
$$

where $I(1)$ denotes the index set of all pairs $(i, j)$ for which the POP condition (4.2) is violated, and $I(2)$ denotes the index set of all quadruples $(i, j, k, l)$ for which the POIP condition (4.3) is violated.

By definition, if the set $I(1)=\varnothing$, then $\operatorname{Alpha}(A, w)=0$. By analogy, if $I(2)=\varnothing$, then $\operatorname{Beta}(A, w)=0$.

Rationale for relaxing the inequalities in (4.2) and (4.3) is this: consider a PC matrix that satisfies all but one condition in (3.1). The one condition that is violated has the form: $a_{k l}>1$, but $w_{k}=w_{l}$. According to relation (3.1), such a matrix is not POP consistent. If substituted into the Alpha index (4.4), then the Alpha would be 0 , which is undesirable, since zero preferential inconsistency should mean consistency. If we transform the strict equality in (3.1) into the non-strict inequality (4.2), then the Alpha index is still equal to 0, but the PC matrix is considered consistent, and the discrepancy vanishes. The same logic applies to relations (3.2) and (4.3). 
Remark 4.3. The differences in parentheses on the right hand side of relations (4.4) and (4.5) are always positive. Thus, the higher is the value of $\operatorname{Alpha}(A, w)$ and $\operatorname{Beta}(A, w)$, the more preferentially inconsistent is a given matrix. Clearly, $\operatorname{Alpha}(A, w) \geq 0$ and $\operatorname{Beta}(A, w) \geq 0$, and $\operatorname{Alpha}(A, w)=0, \operatorname{Beta}(A, w)=0$ if and only if a PC matrix $A$ is (preferentially) consistent. Both indices are not bounded from above.

Remark 4.4. The values of indices Alpha and Beta depend on $w$, a priority vector (vector of weights). There are several methods for the derivation of a priority vector from a PC matrix, the two most common methods are the geometric mean method (GMM) and the eigenvalue method (EVM). These two methods provide identical priority vectors for consistent matrices and slightly different priority vectors in the case of inconsistent matrices of the order $n>3$. In theory, the EVM could be used in Definitions 4.1-4.5 instead of the GMM as well, however, as shown in Csató and Petróczy [27], the EVM does not satisfy an important condition called rank monotonicity, but the GMM does. Further on, the study Mazurek and Kulakowski [49] found that the GMM slightly outperforms the EVM with respect to the satisfaction of the POP and POIP conditions. It should be noted that the eigenvalue method (as a fundamental part of the analytic hierarchy process) was criticised by several prominent researchers in the field, see e.g. Bana e Costa and Vansnick [2], Barzilai [5], or Dyer [30] and suffers several theoretical shortcomings, namely right-left asymmetry [10,36], Pareto inefficiency $[8,9]$, or non-monotonicity [27]. Furthermore, the alternative with the highest priority for all decision-makers is not necessarily the best on the basis of their aggregated preferences [22,53], and the ranking obtained from the principal right eigenvector depends on the choice of the parameter for numerically coded ordinal preferences $[28,32,55]$.

If a decision maker is not interested in the evaluation of the preferential inconsistency of the whole pairwise comparison matrix, but wants to find the worst case of preferential inconsistency instead (recall Koczkodaj's inconsistency index in the numerical consistency framework), indices (4.4) and (4.5) can be appropriately modified:

Definition 4.5. Let $A=\left[a_{i j}\right]$ be a PC matrix, and let $w=\left(w_{1}, \ldots, w_{n}\right)$ be the vector of weights associated with the matrix $A$ via the geometric mean method. Then the preference violation index $\operatorname{Alpha}_{\max }(A, w)$ for the POP condition is given as follows:

$$
\operatorname{Alpha}_{\max }(A, w)=\max \left\{w_{j}-w_{i}\right\}, \quad \forall(i, j) \in I(1) .
$$

Analogously, the preference violation index $\operatorname{Beta}_{\max }(A, w)$ for the POIP condition is given as:

$$
\operatorname{Betamax}_{\max }(A, w)=\max \left\{\frac{w_{k}}{w_{l}}-\frac{w_{i}}{w_{j}}\right\}, \quad \forall(i, j, k, l) \in I(2) .
$$

By definition, if the set $I(1)=\varnothing$, then $\operatorname{Alpha}_{\text {max }}(A, w)=0$. Similarly, if $I(2)=\varnothing$, then $\operatorname{Beta}_{\max }(A, w)=0$.

\section{NumericAl EXAMPLES}

In this section a numerical example is provided to demonstrate the use of the proposed indices.

Example 5.1. Consider the following pairwise comparison matrix $A$ of the order $n=4$ associated with four alternatives:

$$
A=\left[\begin{array}{llll}
1 & 9 & 8 & 6 \\
\frac{1}{9} & 1 & \frac{1}{6} & \frac{1}{2} \\
\frac{1}{8} & 6 & 1 & \frac{1}{3} \\
\frac{1}{6} & 2 & 3 & 1
\end{array}\right] .
$$

The vector of weights derived by the GM method: $w=(0.693,0.047,0.108,0.152)$. 


\section{Check of the POP condition}

$a_{12}=9>1$, so $w_{1}=0.693$ should be greater than or equal to $w_{2}=0.047$, which is satisfied.

$a_{13}=8>1$, so $w_{1}=0.693$ should be greater than or equal to $w_{3}=0.108$, which is satisfied.

$a_{14}=6>1$, so $w_{1}=0.693$ should be greater than or equal to $w_{4}=0.152$, which is satisfied.

$a_{21}=1 / 9<1$, therefore we skip to the next matrix element, and so forth.

Because all 12 individual POP conditions (the POP conditions for each matrix non-diagonal element) are satisfied, the matrix $A$ is $\mathrm{POP}$ consistent, which means that the matrix preserves the order of preferences for all compared objects. The set $I(1)$ is empty, hence $\operatorname{Alpha}(A, w)=0$.

\section{Check of the POIP condition}

$a_{12}=9>1, a_{13}=8>1$, and $a_{12}>a_{13}$ holds, hence, according to (4.3), $w_{1} / w_{2}=14.74$ should be greater than or equal to $w_{1} / w_{3}=6.42$, which is satisfied.

$a_{12}=9>1, a_{14}=6>1$, and $a_{12}>a_{14}$ holds, hence, according to (4.3), $w_{1} / w_{2}=14.74$ should be greater than or equal to $w_{1} / w_{4}=4.56$, which is satisfied.

After checking all 118 remaining cases, four individual violations of the POIP condition can be found, therefore the matrix $A$ is not POIP consistent, which means that the matrix does not preserve intensity of preferences. The set $I(2)=\{(4,3,4,2),(3,2,4,2),(2,4,3,4),(2,4,2,3)\}$.

One can easily check, for instance, that for the quadruple $(4,3,4,2)$ the POIP condition is violated:

$a_{43}=3>1, a_{42}=2>1$, and $a_{43}>a_{42}$ hold, but $w_{4} / w_{3}=1.407$ is less than $w_{4} / w_{2}=3.234$, which contradicts condition (4.3).

The POIP condition for the matrix $A$ is violated in 4 individual cases. Since $\max _{(\mathrm{POP})}=6$ and $\max _{(\mathrm{POIP})}=$ $30, n_{1}=0$ and $n_{2}=4$, VFI attains the following value:

$$
\operatorname{VFI}_{n}(A, w)=\left[\alpha=\frac{n_{1}}{\max _{(\mathrm{POP})}}, \beta=\frac{n_{2}}{\max _{(\mathrm{POIP})}}\right]=[0,2 / 15] .
$$

$\operatorname{Alpha}(A, w)=0$, since $I(1)=\varnothing$.

$\operatorname{Alpha}_{\max }(A, w)=0$, since $I(1)=\varnothing$.

$\operatorname{Beta}(A, w)=\sum_{I(2)}\left(\frac{w_{k}}{w_{l}}-\frac{w_{i}}{w_{j}}\right)=\left(\frac{w_{2}}{w_{3}}-\frac{w_{2}}{w_{4}}\right)+\left(\frac{w_{3}}{w_{4}}-\frac{w_{2}}{w_{4}}\right)+\left(\frac{w_{4}}{w_{2}}-\frac{w_{3}}{w_{2}}\right)+\left(\frac{w_{4}}{w_{2}}-\frac{w_{4}}{w_{3}}\right)=3.279$.

$\operatorname{Beta}_{\max }(A, w)=w_{4} / w_{2}-w_{4} / w_{3}=1.83$.

Example 5.2. Consider the pairwise comparison matrix $A$ of the order $n=4$ from Example 5.1, and let the element $a_{34}$ increase from $1 / 3$ to 175 , while evaluating the Koczkodaj's inconsistency index (KI), see Koczkodaj [38], and the Alpha index in the process. The results are shown in Figure 1. As can be seen, both indices increase monotonically as $a_{34}$ grows, which means the index Alpha satisfies Axiom 4 (see the next section) in this particular case.

\section{AXIOMS FOR PREFERENCE VIOLATION INDICES}

The examination of properties of inconsistency indices attracted many recent studies, see e.g. Alonso and Lamata [1], Brunelli [14,15], Brunelli and Fedrizzi [16], Csató [23,25], Koczkodaj and Urban [41], Mazurek [47], Peláez and Lamata [52]. Their authors call the desirable properties, which inconsistency indices should satisfy, "axioms", and propose their own axiomatic systems. However, all axiomatic systems proposed in the literature are based (directly or indirectly) on standard relation of consistency (2.2). In this section, the axioms from Brunelli and Fedrizzi [16] will be discussed with respect to preference violation indices Alpha and Beta introduced in previous section. 


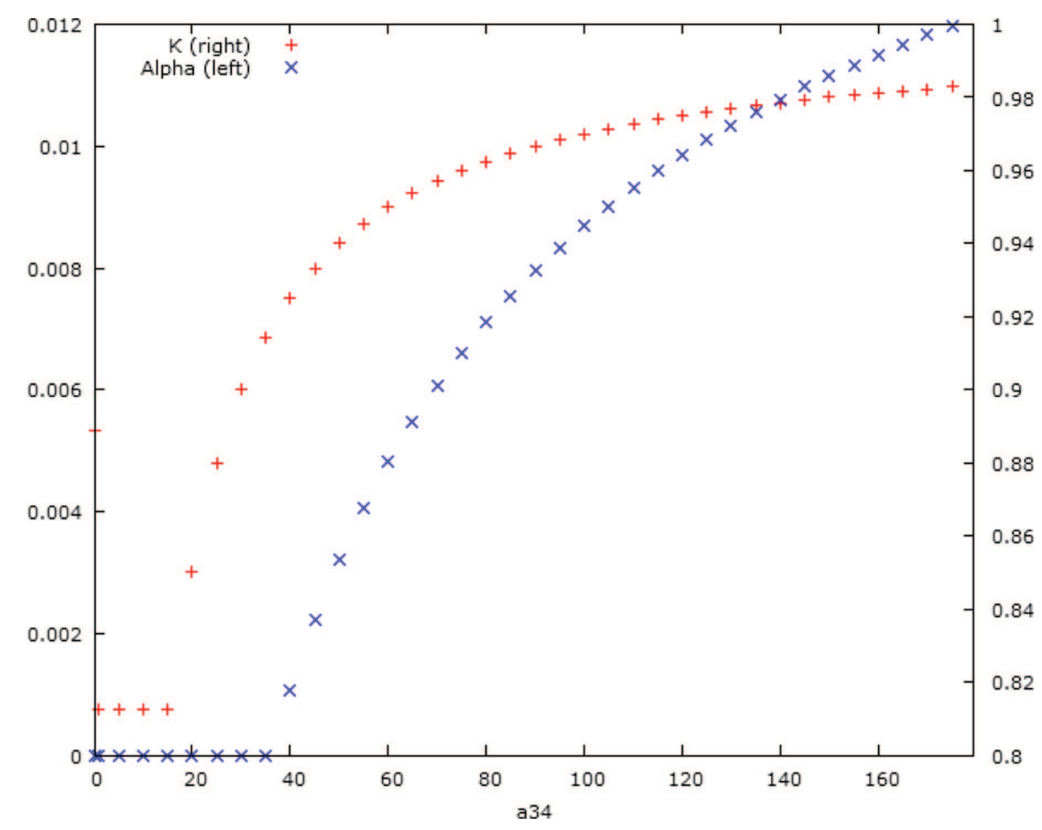

Figure 1. The left scale and blue dots: Alpha; the right scale and red dots: KI.

Axioms proposed in Brunelli and Fedrizzi [16] (adapted):

Let $A$ be a pairwise comparison matrix, let $A^{*}$ denote the set of consistent pairwise comparison matrices, and let $I$ be an inconsistency index.

- Axiom 1 (Existence of a unique element representing consistency).

Formally: an inconsistency index $I$ satisfies Axiom 1 if $A \in A^{*} \Leftrightarrow I(A)=0, \forall A$.

- Axiom 2 (Invariance under permutation of alternatives).

Formally: an inconsistency index $I$ satisfies Axiom 2 if $I(A)=I\left(P A P^{T}\right), \forall A$, where $P$ is a permutation matrix, and $P^{T}$ its transpose.

- Axiom 3 (Monotonicity under reciprocity-preserving mapping).

Formally: let $A(b)=a_{i j}^{b}$. An inconsistency index $I$ satisfies Axiom 3 if $I(A(b)) \geq I(A), \forall b>1, \forall A$.

- Axiom 4 (Monotonicity on single comparisons).

Formally: let $a_{p q} \in A \neq 1$. An inconsistency index $I$ satisfies Axiom 4 if $I\left(a_{p q}(\delta)\right)$ is a non-decreasing function of $\delta, \delta>1$, and $I\left(a_{p q}(\delta)\right)$ is a decreasing function of $\delta, \delta<1, \forall A$.

- Axiom 5 (Continuity).

Formally: an inconsistency index $I$ satisfies Axiom 5 if $I(A)$ is a continuous function of all $a_{i j} \in A, \forall A$.

Study Brunelli [14] introduced an additional axiom:

- Axiom 6 (Invariance under inversion of preferences).

Formally: an inconsistency index $I$ satisfies the Axiom if $I(A)=I\left(A^{T}\right)$, where $A^{T}$ denotes a transposed matrix to $A$.

Since the evaluation of the preferential consistency can be considered a parallel approach to the numerical consistency - inconsistency investigation of pairwise comparison matrices (or judgments in general) with inconsistency indices mentioned in previous sections, also preference violation indices, such as Alpha and Beta proposed in this study, should satisfy some general (desirable) conditions. The question remains, which aforementioned axioms can be adapted for the preferential consistency.

It is shown in Brunelli and Fedrizzi [16] that out of seven examined inconsistency indices, four indices did not satisfy at least one of the aforementioned axioms. This indicates that the set of axioms listed above may be 
too restrictive. Nevertheless, all seven indices satisfied the first two axioms that can be undoubtly considered most crucial to be complied.

Therefore, an axiomatic system in the framework of the preferential consistency should certainly include Axioms 1 and 2 as its core. These two axioms can be adopted without any changes. Also, Axiom 5 can be included, since abrupt changes and "jumps" of the index's values are rather undesirable.

Axioms 3 and 4 were found to be most frequently violated in the Brunelli and Fedrizzi [16] study, moreover, the authors were not able to prove its satisfaction or violation in case of two indices due to non-trivial formulation of the axioms, which indicates that these axioms may not be suitable for theoretical considerations. Whether these two axioms (after appropriate reformulation) should be included into the set of axioms for preferential inconsistency therefore remains an open question and a topic for a discussion.

When constructing an axiomatic system for the preferential consistency, it is important to notice differences between the preferential consistency and the numerical consistency. The latter is based (directly or indirectly) on equation (2.2). The former is based on the set of two inequalities ((4.2) and (4.3)). Where the numerical consistency deals with points as solutions to (2.2), the preferential consistency deals with intersections of intervals in general. That is why the case of the preferential consistency is more complex, which must be taken into account in formulation of an appropriate axiomatic system.

Below, it is shown that both preference violation indices $\operatorname{Alpha}(A, w)$ and $\operatorname{Beta}(A, w)$ satisfy $\operatorname{Axioms} 1,2,5$ and 6 .

Proposition 6.1. Let $A\left[a_{i j}\right]$ be a pairwise comparison matrix, let $w$ denote a priority vector associated with $A\left(a_{i j}\right)$, and let indices $\operatorname{Alpha}(A, w)$ and $\operatorname{Beta}(A, w)$ be defined by relations (4.4) and (4.5), respectively. Then $\operatorname{Alpha}(A, w)$ and $\operatorname{Beta}(A, w)$ satisfy Axiom 1 .

Proof. Let $A$ be the POP consistent: $A\left[a_{i j}\right] \in A^{*}$. Then the index set $I(1)=\varnothing$, hence Alpha $(A, w)=0$. The reverse implication: let $\operatorname{Alpha}(A, w)=0$. Then either: (a) the index set $I(1)$ is empty, hence $A$ is the POP consistent; or (b) the index set $I(1)$ is non-empty, and the sum in (4.4) is zero. However, if for a pair $(i, j)$ is $\left(w_{j}-w_{i}\right)=0$, then the matrix element $a_{i j}$ with corresponding weights $w_{i}, w_{j}$ satisfies relation (4.2), therefore the pair $(i, j)$ does not belong into $I(1)$, which contradicts the initial assumption. Therefore, if Alpha $(A, w)=0$, then necessarily the index set $I(1)$ is empty, and, consequently, the matrix $A$ is the POP consistent.

The proof for the index $\operatorname{Beta}(A, w)$ is analogous.

Proposition 6.2. Let $A\left[a_{i j}\right]$ be a pairwise comparison matrix, and let indices $\operatorname{Alpha}(A, w)$ and $\operatorname{Beta}(A, w)$ be defined by relations (4.4) and (4.5), respectively. Then $\operatorname{Alpha}(A, w)$ and $\operatorname{Beta}(A, w)$ satisfy Axiom 2.

Proof. It is obvious since both indices $\operatorname{Alpha}(A, w)$ and $\operatorname{Beta}(A, w)$ are invariant under a change of the order (renaming) of compared objects.

Proposition 6.3. Let $A\left[a_{i j}\right]$ be a pairwise comparison matrix, and let indices $\operatorname{Alpha}(A, w)$ and $\operatorname{Beta}(A, w)$ be defined by relations (4.4) and (4.5), respectively. Then $\operatorname{Alpha}(A, w)$ and $\operatorname{Beta}(A, w)$ satisfy Axiom 5.

Proof. Since $w_{i} \neq 0, \forall i$, all fractions in $\operatorname{Alpha}(A, w)$ and $\operatorname{Beta}(A, w)$ are well defined and continuous, and a finite sum of continuous functions is also continuous.

Proposition 6.4. Let $A\left[a_{i j}\right]$ be a pairwise comparison matrix, and let indices $\operatorname{Alpha}(A, w)$ and $\operatorname{Beta}(A, w)$ be defined by relations (4.4) and (4.5), respectively. Then $\operatorname{Alpha}(A, w)$ and $\operatorname{Beta}(A, w)$ satisfy Axiom 6.

Proof. It is obvious.

Proposition 6.5. Let $A\left[a_{i j}\right]$ be a pairwise comparison matrix, and let indices $\operatorname{Alpha}_{\max }(A, w)$ and $\operatorname{Beta}_{\max }(A, w)$ be defined by relations (4.6) and (4.7), respectively. Then $\operatorname{Alpha}_{\max }(A, w)$ and $\operatorname{Beta}_{\max }(A, w)$ satisfy Axioms 1, 2, 5 and 6. 
Proof. It is obvious.

The following proposition states that when a pairwise comparison matrix $A$ is the COP consistent and all preferences are abided, but intensified by a factor $b>1$, then the matrix $A$ remains the COP consistent (the feature also satisfied by numerically consistent matrices), if the priority deriving method is the geometric mean method, which is due to the scale invariance of the GMM, see Csató [21,23].

Proposition 6.6. Let $A\left[a_{i j}\right]$ be a $n \times n$ pairwise comparison matrix, let $w$ denote the priority vector associated with $A\left[a_{i j}\right]$ by the $G M$ method, and let indices $\operatorname{Alpha}(A, w)$ and $\operatorname{Beta}(A, w)$ be defined by relations (4.6) and (4.7), respectively. Further, let $A\left[a_{i j}\right]$ be the COP consistent, hence $\operatorname{Alpha}(A, w)=0$ and $\operatorname{Beta}(A, w)=0$. Let $A^{(b)}\left[a_{i j}\right]$ be the matrix derived from $A\left[a_{i j}\right]$ by raising each element $a_{i j}$ of $A$ to the bth power, where $b>1$. Then, $A^{(b)}\left[a_{i j}\right]$ is also the COP consistent.

Proof. The matrix $A\left[a_{i j}\right]$ is the COP consistent, hence if $a_{i j}>1$, then $w_{i} \geq w_{j}, \forall i, j \in\{1, \ldots, n\}$, and according to $(2.3), w_{i}=\left(\prod_{k=1}^{k=n} a_{i k}\right)^{1 / n} \geq w_{j}=\left(\prod_{k=1}^{k=n} a_{j k}\right)^{1 / n}$, therefore $\prod_{k=1}^{k=n} a_{i k} \geq \prod_{k=1}^{k=n} a_{j k}$. After raising each $a_{i j}$ to the $b$ th power $(b>1)$, we obtain: $w_{i}^{(b)}=\left(\prod_{k=1}^{k=n} a_{i k}^{b}\right)^{1 / n} \geq w_{j}^{(b)}=\left(\prod_{k=1}^{k=n} a_{j k}^{b}\right)^{1 / n}$, since $\left(\prod_{k=1}^{k=n} a_{i k}\right)^{b / n} \geq$ $\left(\prod_{k=1}^{k=n} a_{j k}\right)^{b / n}$, and after extracting the root we get: $\prod_{k=1}^{k=n} a_{i k} \geq \prod_{k=1}^{k=n} a_{j k}$, which is true according to the initial assumption.

Studies Koczkodaj et al. [43] and Mazurek [48] argue that an inconsistency index should be normalized to an $[0,1]$ interval, or, at least, to be bounded from above. The next proposition states that both preference violation indices $\operatorname{Alpha}(A, w)$ and $\operatorname{Beta}(A, w)$ are bounded from above.

Proposition 6.7. Let $A\left[a_{i j}\right]$ be a pairwise comparison matrix of order $n$, and let indices $\operatorname{Alpha}(A, w)$ and $\operatorname{Beta}(A, w)$ be defined by relations (4.6) and (4.7), respectively. Then both $\operatorname{Alpha}(A, w)$ and $\operatorname{Beta}(A, w)$ are bounded from above.

Proof. Both $\operatorname{Alpha}(A, w)$ and $\operatorname{Beta}(A, w)$ are continuous functions and the set $w_{i}$, such that $0<w_{i}<1, \forall i$, constitutes their domain. Since the domain set of both (continuous) functions is bounded, also their range is bounded.

The following proposition provides an upper bound estimate for the index $\operatorname{Alpha}(A, w)$ with respect to the matrix size $n$, which can be used for a normalized version of the index.

Proposition 6.8. Let $A\left[a_{i j}\right]$ be a pairwise comparison matrix of order $n$, and let $\operatorname{Alpha}(A, w)$ be defined by relation (4.6). Then $\operatorname{Alpha}(A, w)<\left(n^{2}-n\right) / 2$.

Proof. Index $\operatorname{Alpha}(A, w)$ is defined as the sum of terms $\left(w_{j}-w_{i}\right)$ such that $w_{j}>w_{i}$, where $i, j \in I(2)$. This sum contains up to $\left(n^{2}-n\right) / 2$ terms corresponding to the maximum number of POP violations. Since $\left(w_{j}-w_{i}\right)<1, \forall i, j \in I(2)$, we get: $\operatorname{Alpha}(A, w)=\sum_{I(1)}\left(w_{j}-w_{i}\right)<\left(n^{2}-n\right) / 2 \cdot 1=\left(n^{2}-n\right) / 2$.

Due to fractions in the definition of the index Beta, the index is bounded from above only when a pairwise comparison scale is bounded from above.

Proposition 6.9. Let $A\left[a_{i j}\right]$ be a pairwise comparison matrix of the order $n$, and let $\operatorname{Beta}(A, w)$ be defined by relation (4.5). Let $a_{i j} \leq K, \forall i, j$. Then $\operatorname{Beta}(A, w)$ is bounded from above.

Proof. It is sufficient to show that fractions in the definitions of $\operatorname{Beta}(A, w)$ are bounded. Without loss of generality assume that $a_{1 j}=K, \forall j, 1<j \leq n$ and $a_{2 j}=1 / K, \forall 1<j \leq n, j \neq 2$, so that the fraction $w_{1} / w_{2}$ is maximal possible. We show that it is bounded from above. From the GM method it follows that $w_{1}=K^{\frac{n-1}{n}}$ and $w_{2}=K^{\frac{1-n}{n}}$. Therefore, $w_{1} / w_{2}=K^{\frac{n-1}{n}} / K^{\frac{1-n}{n}}=K^{2 n-2}$. As a consequence, $\operatorname{Beta}(A, w)<$ $\frac{1}{4}\left(n^{2}-n\right)\left(n^{2}-n-2\right) K^{2 n-2}$. 
TABle 1. Percentile values for Alpha and Beta, $n=4$.

\begin{tabular}{llllll}
\hline \hline Percentile & Alpha & Beta & Percentile & Alpha & Beta \\
\hline 0 & 0 & 0 & 55 & 0.074 & 16.62 \\
5 & 0 & 3.95 & 60 & 0.106 & 17.89 \\
10 & 0 & 6.86 & 65 & 0.144 & 19.35 \\
15 & 0 & 8.48 & 70 & 0.179 & 21.07 \\
20 & 0 & 9.76 & 75 & 0.208 & 23.03 \\
25 & 0 & 10.76 & 80 & 0.233 & 25.18 \\
30 & 0 & 11.71 & 85 & 0.257 & 28.47 \\
35 & 0 & 12.69 & 90 & 0.282 & 33.27 \\
40 & 0 & 13.57 & 95 & 0.316 & 41.32 \\
45 & 0.024 & 14.44 & 100 & 0.415 & 107.36 \\
50 & 0.046 & 15.48 & & & \\
\hline
\end{tabular}

Whether the preference violation indices $\operatorname{Alpha}(A, w)$ and $\operatorname{Beta}(A, w)$, or $\operatorname{Alpha}_{\max }(A, w)$ and $\operatorname{Beta}_{\max }(A, w)$, respectively, satisfy or violate Axioms 3 and 4 remains unknown (see Example 5.2 where Axiom 4 is satisfied by Alpha), but a numerical study that is going to follow the presented research will hopefully find the answers. However, it should be noted that Axioms 1, 2,5 and 6 are general - they do not depend on a type of inconsistency. On the other hand, Axioms 3 and 4 assume the notion of (in)consistency given by relation (2.2), and state what should happen when a single matrix element deviates from (2.2). It is clear that such axioms are not particularly suitable for Alpha and Beta indices, since, for instance, the value of Beta depends on an interplay of two matrix elements and four weights in each parenthesis within the sum in relation (4.5). Hence, Axioms 3 and 4 might need a modification.

Finally, although the preference violation indices were introduced in the theoretical framework of multiplicative pairwise comparisons, the indices can be easily reformulated for other pairwise comparison systems, e.g. fuzzy or additive, since all these systems form one Abelian linearly ordered group, and can be mapped one to another by appropriate isomorphisms, see e.g. Cavallo and D'Apuzzo [18], or Kulakowski et al. [46].

\section{Monte Carlo simulations for $n=4$}

In this section values of Alpha and Beta indices were examined for 5000 randomly generated multiplicative pairwise comparisons matrices of order $n=4$, where matrix elements were drawn from Saaty's fundamental scale $\{1 / 9,1 / 8, \ldots, 1, \ldots, 8,9\}$, to provide the first insight into behavior of both inconsistency indices. The priority vector of each matrix was calculated via the geometric mean method. The random generation of matrix entries above the main diagonal was performed in $R$ with the use of the uniform distribution, and entries below the main diagonal were calculated as their reciprocals.

Out of 1000 generated matrices, $41.2 \%$ of matrices had Alpha $=0$, hence they were POP consistent, and $0.9 \%$ had Beta $=0$, hence they were POIP consistent. The frequency distribution of Alpha and Beta values is shown in Figures 2 and 3. Pearson's correlation coefficient between Koczkodaj's inconsistency index KI and Alpha was 0.497 and between KI and Beta 0.135. Table 1 provides percentiles for Alpha and Beta values. Median values for both indices were found as follows: $x_{\text {med }}$ (Alpha) $=0.046$ and $x_{\text {med }}($ Beta $)=15.48$.

In the future, Monte Carlo simulations for $n>4$ are going to be performed, but it must be noted that the number of POIP conditions that have to be checked grows rapidly with increasing $n$, making simulations particularly difficult and time consuming (in the case of $n=7$, for example, 1680 individual POIP conditions must be checked just for one generated matrix!). 


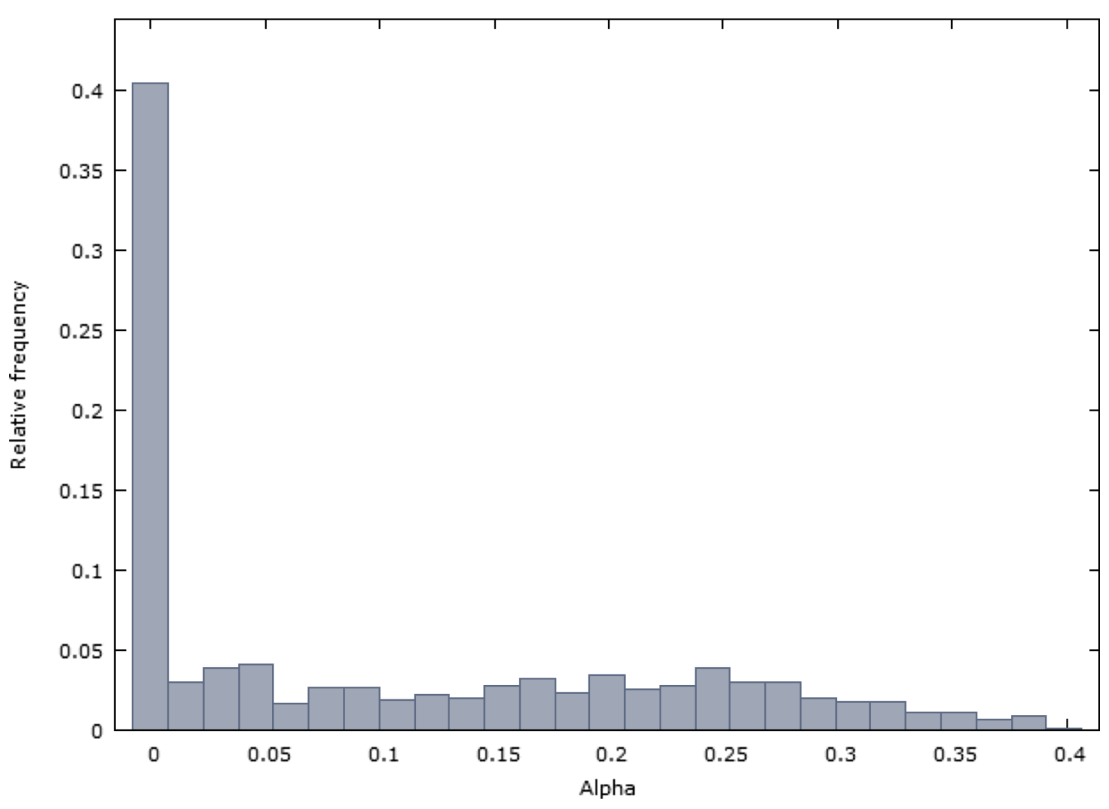

FIGURE 2. Frequency distribution of Alpha.

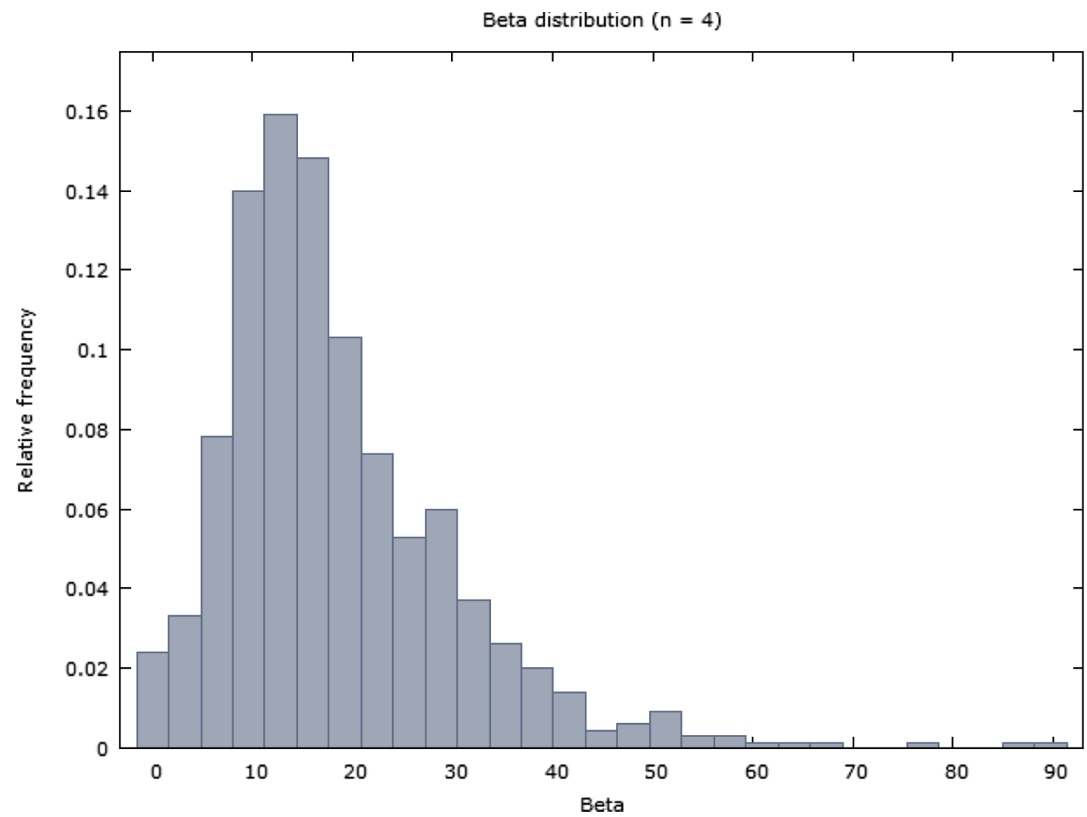

FigURE 3. Frequency distribution of Beta. 


\section{Conclusions}

The aim of this paper was to introduce preference violation indices for the condition of the order preservation (COP) in multiplicative pairwise comparisons. While index VFI provides a frequency of violations of the COP condition, other two indices, Alpha and Beta, aim at measuring the deviation of pairwise comparisons (with an associated priority vector) from the "preferential consistency" formulated by Bana e Costa and Vansnick [2]. Also, an axiomatic system for the proposed preference violation indices was discussed, and it was shown that both indices Alpha and Beta satisfy uniqueness, invariance under permutation, invariance under inversion of preferences and continuity axioms. Further study may focus on the relationship between newly introduced indices Alpha and Beta, and standard inconsistency indices such as CI, CR, or KI, or generalization of the proposed indices into fuzzy or alo-groups frameworks. The evaluation of the COP condition for incomplete matrices can be considered as well.

Acknowledgements. The research was supported by the project GACR Nr. 21-03085S.

\section{REFERENCES}

[1] J.A. Alonso and M.T. Lamata, Consistency in the analytic hierarchy process: a new approach. Int. J. Uncertainty Fuzziness Knowl. Based Syst. 14 (2006) 445-459.

[2] C.A. Bana e Costa and J. Vansnick, A critical analysis of the eigenvalue method used to derive priorities in AHP. Eur. J. Oper. Res. 187 (2008) 1422-1428.

[3] J. Barzilai, Deriving weights from pairwise comparison matrices. J. Oper. Res. Soc. 48 (1997) 1226-1232.

[4] J. Barzilai, Consistency measures for pairwise comparison matrices. J. Multi-Criteria Decis. Anal. 7 (1998) $123-132$.

[5] J. Barzilai, On the decomposition of value functions. Oper. Res. Lett. 22 (1998) 159-170.

[6] J. Barzilai, W.D. Cook and B. Golany, Consistent weights for judgements matrices of the relative importance of alternatives. Oper. Res. Lett. 6 (1987) 131-134.

[7] M. Behzadian, R.B. Kazemzadeh, A. Albadvi and M. Aghdasi, PROMETHEE: a comprehensive literature review on methodologies and applications. Eur. J. Oper. Res. 200 (2010) 198-215.

[8] R. Blanquero, E. Carrizosa and E. Conde, Inferring efficient weights from pairwise comparison matrices. Math. Methods Oper. Res. 64 (2006) 271-284.

[9] S. Bozóki, Inefficient weights from pairwise comparison matrices with arbitrarily small inconsistency. Optimization 63 (2014) 1893-1901.

[10] S. Bozóki and T. Rapcsák, On Saaty's and Koczkodaj's inconsistencies of pairwise comparison matrices. J. Global Optim. 42 (2008) 157-175.

[11] S. Bozóki, L. Dezső, A. Poesz and J. Temesi, Analysis of pairwise comparison matrices: an empirical research. Ann. Oper. Res. 211 (2013) 511-528.

[12] S. Bozóki, L. Csató and J. Temesi, An application of incomplete pairwise comparison matrices for ranking top tennis players. Eur. J. Oper. Res. 248 (2016) 211-218.

[13] M. Brunelli, Recent advances on inconsistency indices for pairwise comparisons - a commentary. Fundam. Inf. 144 (2016) $321-332$.

[14] M. Brunelli, Studying a set of properties of inconsistency indices for pairwise comparisons. Ann. Oper. Res. 248 (2017) $143-161$.

[15] M. Brunelli, A survey of inconsistency indices for pairwise comparisons. Int. J. General Syst. 47 (2018) 751-771.

[16] M. Brunelli and M. Fedrizzi, Axiomatic properties of inconsistency indices for pairwise comparisons. J. Oper. Res. Soc. 66 (2015) 1-15.

[17] M. Brunelli, L. Canal and M. Fedrizzi, Inconsistency indices for pairwise comparison matrices: a numerical study. Ann. Oper. Res. 211 (2013) 493-509.

[18] B. Cavallo and L. D'Apuzzo, Preservation of preferences intensity of an inconsistent Pairwise Comparison Matrix. Int. J. Approximate Reasoning 116 (2020) 33-42.

[19] X. Chao, G. Kou, T. Li and Y. Peng, Jie Ke versus AlphaGo: a ranking approach using decision making method for large-scale data with incomplete information. Eur. J. Oper. Res. 265 (2018) 239-247.

[20] L. Csató, Ranking by pairwise comparisons for Swiss-system tournaments. Cent. Eur. J. Oper. Res. 21 (2013) $783-803$.

[21] L. Csató, On the ranking of a Swiss system chess team tournament. Ann. Oper. Res. 254 (2017) 17-36.

[22] L. Csató, Eigenvector method and rank reversal in group decision making revisited. Fundam. Inf. 156 (2017) $169-178$.

[23] L. Csató, Characterization of the row geometric mean ranking with a group consensus axiom. Group Decis. Negotiation $\mathbf{2 7}$ (2018) 1011-1027.

[24] L. Csató, Characterization of an inconsistency measure for pairwise comparison matrices. Ann. Oper. Res. 261 (2018) $155-165$.

[25] L. Csató, Axiomatizations of inconsistency indices for triads. Ann. Oper. Res. 280 (2019) 99-110. 
[26] L. Csató, A characterization of the Logarithmic Least Squares Method. Eur. J. Oper. Res. 276 (2019) $212-216$.

[27] L. Csató and D.G. Petróczy, On the monotonicity of the eigenvector method. Eur. J. Oper. Res. 292 (2021) $230-237$.

[28] L. Csató and L. Rónyai, Incomplete pairwise comparison matrices and weighting methods. Fundam. Inf. 144 (2016) 309-320.

[29] L. Csató and C. Tóth, University rankings from the revealed preferences of the applicants. Eur. J. Oper. Res. 286 (2020) 309-320.

[30] J.S. Dyer, Remarks on the analytic hierarchy process. Manage. Sci. 36 (1990) 249-258.

[31] J. Fichtner, On deriving priority vectors from matrices of pairwise comparisons. Soc.-Econ. Planning Sci. 20 (1986) 341-345.

[32] C. Genest, F. Lapointe and S.W. Drury, On a proposal of Jensen for the analysis of ordinal pairwise preferences using Saaty's eigenvector scaling method. J. Math. Psychol. 37 (1993) 575-610.

[33] B. Golden and Q. Wang, An alternate measure of consistency. In: The Analytic Hierarchy Process, Applications and Studies, edited by B. Golden, E. Wasil and P.T. Harker, Springer-Verlag, Berlin-Heidelberg (1989) 68-81.

[34] K. Govindan and M.B. Jepsen, ELECTRE: a comprehensive literature review on methodologies and applications. Eur. J. Oper. Res. 250 (2015) 1-29.

[35] W. Holsztynski and W.W. Koczkodaj, Convergence of inconsistency algorithms for the pairwise comparisons. Inf. Process. Lett. 59 (1996) 197-202.

[36] C.R. Johnson, W.B. Beine and T.J. Wang, Right-left asymmetry in an eigenvector ranking procedure. J. Math. Psychol. 19 (1979) 61-64.

[37] P.T. Kazibudzki, An examination of performance relations among selected consistency measures for simulated pairwise judgments. Ann. Oper. Res. 244 (2016) 525-544.

[38] W.W. Koczkodaj, A new definition of consistency of pairwise comparisons. Math. Comput. Modell. 18 (1993) $79-84$.

[39] W.W. Koczkodaj, Statistically accurate evidence of improved error rate by pairwise comparisons. Perceptual Motor Skills $\mathbf{8 2}$ (1996) 43-48.

[40] W. Koczkodaj and R. Szwarc, On axiomatization of inconsistency indicators for pairwise comparisons. Fundam. Inf. 132 (2014) 485-500.

[41] W.W. Koczkodaj and R. Urban, Axiomatization of inconsistency indicators for pairwise comparisons. Int. J. Approximate Reasoning 94 (2018) 18-29.

[42] W.W. Koczkodaj, L. Mikhailov, G. Redlarski, M. Soltys, J. Szybowski, G. Tamazian, E. Wajch and K.K.F. Yuen, Important facts and observations about pairwise comparisons (the special issue edition). Fundam. Inf. 144 (2016) 291-307.

[43] W.W. Koczkodaj, J.-P. Magnot, J. Mazurek, J.F. Peters, H. Rakhshani, M. Soltys, D. Strzalka, J. Szybowski and A. Tozzi, On normalization of inconsistency indicators in pairwise comparisons. Int. J. Approximate Reasoning 86 (2017) 73-79.

[44] K. Kulakowski, Notes on order preservation and consistency in AHP. Eur. J. Oper. Res. 245 (2015) $333-337$.

[45] K. Kulakowski and D. Talaga, Inconsistency indices for incomplete pairwise comparisons matrices. Int. J. General Syst. 49 (2020) 174-200.

[46] K. Kulakowski, J. Mazurek, J. Ramík and M. Soltys, When is the condition of order preservation met? Eur. J. Oper. Res. 277 (2019) 248-254.

[47] J. Mazurek, Some notes on the properties of inconsistency indices in pairwise comparisons. Oper. Res. Decis. 28 (2018) $27-42$.

[48] J. Mazurek, On the problem of different pairwise comparison scales in the multiplicative AHP framework. Sci. Papers Univ. Pardubice 46 (2019) 124-133.

[49] J. Mazurek and K. Kulakowski, Satisfaction of the condition of order preservation: a simulation study. Operations Research and Decisions 2 (2020) 77-89.

[50] J. Mazurek and R. Perzina, On the inconsistency of pairwise comparisons: an experimental study. Sci. Papers Univ. Pardubice 24 (2017) 102-109.

[51] J. Mazurek and J. Ramík, Some new properties of inconsistent pairwise comparison matrices. Int. J. Approximate Reasoning 113 (2019) 119-132.

[52] J.I. Peláez and M.T. Lamata, A new measure of inconsistency for positive reciprocal matrices. Comput. Math. App. 46 (2003) 1839-1845.

[53] J. Pérez and E. Mokotoff, Eigenvector priority function causes strong rank reversal in group decision making. Fundam. Inf. 144 (2016) 255-261.

[54] D.G. Petróczy, An alternative quality of life ranking on the basis of remittances. Soc.-Econ. Planning Sci. 78 (2021) 101042.

[55] D.G. Petróczy and L. Csató, Revenue allocation in Formula One: a pairwise comparison approach. Int. J. General Syst. 50 (2021) 243-261.

[56] J. Ramík, Pairwise comparison matrix with fuzzy elements on alo-groups. Inf. Sci. 297 (2015) $236-253$.

[57] T.L. Saaty, A scaling method for priorities in hierarchical structures. J. Math. Psychol. 15 (1977) 234-281.

[58] T.L. Saaty, The Analytic Hierarchy Process. McGraw-Hill, New York (1980). 
[59] T.L. Saaty, Decision making - the analytic hierarchy and network processes (AHP/ANP). J. Syst. Sci. Syst. Eng. 13 (2004) $1-35$.

[60] O.S. Vaidya and S. Kumar, Analytic hierarchy process: an overview of applications. Eur. J. Oper. Res. 169 (2006) 1-29.

\section{Subscribe to Open (S2O) A fair and sustainable open access model}

This journal is currently published in open access under a Subscribe-to-Open model (S2O). S2O is a transformative model that aims to move subscription journals to open access. Open access is the free, immediate, online availability of research articles combined with the rights to use these articles fully in the digital environment. We are thankful to our subscribers and sponsors for making it possible to publish this journal in open access, free of charge for authors.

\section{Please help to maintain this journal in open access!}

Check that your library subscribes to the journal, or make a personal donation to the S2O programme, by contacting subscribers@edpsciences.org

More information, including a list of sponsors and a financial transparency report, available at: https://www. edpsciences.org/en/maths-s2o-programme 